\title{
Robust Fluidic Biocompatible Strain Sensor Based on PEDOT:PSS/CNT Composite for Human-wearable and High-end Robotic Applications
}

\author{
Faiza Jabbar, ${ }^{1 \dagger}$ Afaque Manzoor Soomro, ${ }^{1,2 \dagger}$ Jae-wook Lee, ${ }^{1}$ Muhsin Ali, ${ }^{1}$ \\ Young Su Kim, ${ }^{1}$ Sang-ho Lee, ${ }^{3}$ and Kyung Hyun Choi ${ }^{1 *}$ \\ ${ }^{1}$ Department of Mechatronics Engineering, Jeju National University, 63243, Republic of Korea \\ ${ }^{2}$ Department of Electrical Engineering, Sukkur IBA University, Airport Road, 56200, Pakistan \\ ${ }^{3}$ Bio PD, Office of Strategic R\&D Planning, 305, Teheran-ro, Gangnam-gu, Seoul, Republic of Korea
}

(Received September 9, 2020; accepted October 23, 2020)

Keywords: fluidic strain sensor, robotics, wearable electronics, biocompatibility

A robust fluidic strain sensor based on a biocompatible conductive poly(3,4-ethylenedioxythiophene) polystyrene sulfonate multiwall carbon nanotube (PEDOT:PSS/MWCNT) liquid is proposed. A simple fabrication process is followed by the 3D printing of a 2.5 cycle sinusoidal channel, and the infiltration liquid is infilled using custommade automated pumping syringes. The sensor showed a highly linear response $\left(R^{2}=0.9935\right)$, a minimal hysteresis $(1.56 \%$ ), a gauge factor $(G F)$ of 89.4 , and a two-order change in resistance up to $150 \%$ strain. The device was tested for 1000 cycles at $30 \%$ strain and showed the ability to follow the applied strain up to a maximum frequency of $10 \mathrm{~Hz}$. Moreover, the device showed a significantly stable response under the full humidity range $(0-100 \% \mathrm{RH})$ and at temperatures from 20 to $40{ }^{\circ} \mathrm{C}$. As a proof-of-concept demonstration, the fabricated sensor was used with a robotic leg as a high-end feedback sensor. Furthermore, the biocompatibility of the device was confirmed before its applications in wearable electronics by the cell viability assessment of human epidermal keratinocytes (HEKp) and human umbilical cord vascular endothelial cells (HUVEC). The simple fabrication process and ultrahigh sensitivity of the device make it a robust candidate for wearable and robotic applications.

\section{Introduction}

Over the past few years, flexible electronics has significantly captured researchers' attention owing to its myriad applications, including physiological monitoring, ${ }^{(1-3)}$ wearable electronics, ${ }^{(4-6)}$ medical devices, protheses, smart clothes, and soft robotics. ${ }^{(7-9)}$ In flexible devices, soft strain sensors play a major role owing to their vibrant features, such as high compliance, low weight, long lifetime, low cost, and the ability to sense the complex movements of soft robots and human body parts. ${ }^{(10)}$ Unlike conventional sensors with rigid electronic subsystems and a limited sensing range, the robust design structure of soft sensors makes them ideal as an integral part of such high-end feedback applications. ${ }^{(1,12)}$

\footnotetext{
${ }^{\dagger}$ Authors contributed equally.

*Corresponding author: e-mail: amm@jejunu.ac.kr

https://doi.org/10.18494/SAM.2020.3085
} 
Strain sensors have gained significant importance in fields such as human health monitoring, hand and limb movement detection, blood pulse monitoring, respiration rate monitoring, feedback-enabled soft robots, flexible touch screens, and electronic skin. Conventional strain sensors utilize a mature technology by exploiting various buckling, meshing, and interlocking films and foams in graphene and carbon. ${ }^{(13,14)}$ In addition, inorganic conductive materials, which contain silver, gold, and $\mathrm{ZnO}$ nanowires, have also been explored. ${ }^{(15,16)}$ In such sensors, the fundamental working mechanism is based on the transformation of conductive networks along with the elongation of an elastic material. ${ }^{(17,18)}$ These sensors have high sensitivity changes in strain; however, this not only makes the fabrication process complex but also results in large hysteresis, which makes the measurement process less precise. To resolve this problem, percolation networks of inorganic materials have been used to develop piezocapactitance-based strain sensors. These sensors have improved linearity and less hysteresis but lack sensitivity with a gauge factor $(G F)$ (in the range of 1.0 or less). ${ }^{(19,20)}$

Recently, soft strain sensors have been developed using conductive liquids. These sensors have the advantages of a compliant nature, low cost, and high suitability of mass production, which allow them to efficiently operate in soft electronics applications. Conventional fluidic sensors are fabricated using three types of conductive liquids, electrolytes based on salts, such as KCl-glycerol, ionic liquids, and liquid metals. ${ }^{(21-23)}$ Moreover, the working principle of such sensors is based on changing electrical resistance due to the changing length of the liquid path caused by stretching, bending, and other forms of deformation. ${ }^{(24)}$ Conductive carbon greases have also been reported in the literature; however, in such a scenario, shear has a significant effect on the resistivity of conductive solutions; thus, they have a large memory effect owing to their viscoelasticity attributes. Moreover, researchers have explored eutectic gallium-indium alloy (EGaIn) and GaInSn liquid metals to fabricate strain sensors. In fact, these liquids have a low melting point and a good electrical conductivity of 3.4-3.5 $\times 10^{6} \mathrm{~S} \mathrm{~m}^{-1}$, and have been used in different robotic and human motion monitoring applications. ${ }^{(25)}$ However, the main hurdle to their mass production is their high cost. Hence, work is still needed to realize a fluidic strain sensor that can achieve a robust blend of features such as less hysteresis, simple design structure, fast response and recovery time, large strain sensing range, and most importantly, biocompatibility, which is an indispensable requirement for the risk-free usage of sensors for health monitoring. ${ }^{(26)}$ In this study, we have proposed a fluidic strain sensor based on a sinusoidal pattern filled with a novel biocompatible conductive poly(3,4-ethylenedioxythiophene) polystyrene sulfonate multiwall carbon nanotube (PEDOT:PSS/MWCNT) composite liquid. The presence of MWCNTs further increases the conductivity of PEDOT:PSS solution. A twoorder change in electrical resistance was observed across the electrodes of the developed sensor upon applying uniaxial strain of up to $150 \%$. The sensor is fabricated using a simple process and exhibits high performance with a hysteresis of $1.56 \%$, considerably less than that of existing biocompatible solutions in addition to excellent sensitivity with a maximum $G F$ of 89.4 at $150 \%$ strain. Moreover, the as-developed sensor achieves high linearity $\left(R^{2}=0.9935\right)$, stable cyclic performance for 1000 cycles at $30 \%$ strain, and the ability to follow the applied strain up to a maximum speed of $10 \mathrm{~Hz}$. Moreover, the structure of the sensor is based on a sinusoidal pattern with 2.5 cycles, which was simulated in our previous study. ${ }^{(5)}$ To confirm the device 
performance under various environmental conditions, it was tested under the full humidity range $(0-100 \% \mathrm{RH})$ at various temperatures $\left(20-40{ }^{\circ} \mathrm{C}\right)$ using our in-house built electronically controlled chamber. As a proof-of-concept demonstration, the fabricated sensor was used with a single joint robotic leg as a high-end feedback sensor. Moreover, prior to using the sensor as a wearable sensor, the biocompatibility of the device was tested by the cell viability assessment of human epidermal keratinocytes (HEKp) and human umbilical cord vascular endothelial cells (HUVEC). The simple fabrication process and ultrahigh sensitivity of the device make it a robust candidate for wearable and robotic applications.

\section{Methods}

\subsection{Materials}

MWCNT ( $>98 \%$ carbon basis, O.D. $6-13 \mathrm{~nm}, \mathrm{~L} \quad 2.5-20 \mu \mathrm{m})$ and PEDOT:PSS ink (3.0-4.0 wt \%) were purchased from Sigma Aldrich. Dimethylformamide (DMF) solvent was acquired from Alfa Aesar. For the matrix to hold the conductive liquid through the sinusoidal channel, Ecoflex 00-30 (Young's modulus $70 \mathrm{kPa}$ ) purchased from Smooth-on was used. First, different dispersions with MCWNT concentrations ranging from 0.10 to $0.3 \mathrm{wt} \%$ in $\mathrm{DMF}$ solvent were prepared. However, on the basis of repeated experiments, $50 \mathrm{mg}$ of MWCNT in $25 \mathrm{ml}$ of DMF was used, which was ultrasonicated for $0.5 \mathrm{~h}$ to ensure that the MWCNT had completely dispersed. To make the composite, PEDOT:PSS (3.0-4.0 wt $\%$ ) was poured into the MWCNT solution in a ratio of 2:1, which was ultrasonicated for $2.5 \mathrm{~h}$. Finally, the PEDOT:PSS/MWCNT polymer composite was centrifuged at $1200 \mathrm{rpm}$ for $20 \mathrm{~min}$ to separate large bundles, which significantly affect the homogeneity of the solution. This process was repeated until a clear solution was obtained.

\subsection{Fabrication of sensor}

The fabrication of the fluidic strain sensor followed a two-step process. In the first step, an ultrastretchable elastomer, Ecoflex 00-30, was used. This material is commercially provided in two parts, $\mathrm{A}$ and $\mathrm{B}$, which are mixed in an equal weight ratio (1:1). Prior to designing the elastomeric matrix, a mold containing a sinusoidal pattern was modeled using SolidWorks 13.0 and then 3D-printed using a Creality CR20 printer by fused deposition modeling (FDM) as in our previous study. ${ }^{(5)}$ The prepared Ecoflex solution was first mixed for $1 \mathrm{~h}$, stirred, and then degassed. Before pouring the Ecoflex solution into the printed mold, two siliconcoated electrodes were attached. The solution present in the mold was cured for more than $4 \mathrm{~h}$ until it was fully cured, after which, it was carefully peeled off with great care so that the engraved channel was not damaged. A stable connection between the electrodes and the conductive liquid, and similarly with the measurement electronics, was of great importance, as a large strain such as $150 \%$ could break the electrical connection easily; to resolve this problem, silicon-coated wires were used. This enabled the sensor to have a strong soft-to-rigid connection, improved the compatibility with the elastomeric matrix, and had a minimum effect 
on the sensor readouts. Moreover, the thickness variation was made in relation to gradient nature in the close vicinity of the electrodes; this further helped significantly improve the sensor dimensional stability in the case of high-frequency strain applications. For the encapsulation of the sensor pattern, a thin membrane was prepared by blade coating. The membrane was halfcured, which took around $2 \mathrm{~h}$ at room temperature, and then it was bonded with an elastomeric matrix with a sinusoidal pattern. The pattern was fully encapsulated after bonding for $2 \mathrm{~h}$. Moreover, the thickness of the membrane was kept in the range of $0.8-1.0 \mathrm{~mm}$.

In the second step, the channel was filled with the liquid. However, with manual handling, two syringes from both ends could not fill the channel completely, leaving many bubbles that severely degraded the sensor sensing performance. Thus, a high synchronization between the two syringes was required, which was not possible with human handling. To make this possible, the syringes were actuated by stepper motors, which were controlled by an Arduino Mega. A program in $\mathrm{C}++$ language was used to further calibrate the process. As a result, the conductive liquid was infilled using an automated process, and no bubbles were formed. However, owing to the filling of the channel, two tiny holes, which were closed using epoxy glue, were created. Figure 1 shows the sensor fabrication steps.

The dimensions of the proposed sensor are the same as in our previous study. ${ }^{(5)}$ Briefly, the height and half-cycle (total of 2.5 cycles) width of the sinusoidal pattern are 12 and $10 \mathrm{~mm}$, respectively. Moreover, the sensor area is $54 \times 20 \mathrm{~mm}$, which can be seen in Fig. S1. The fully

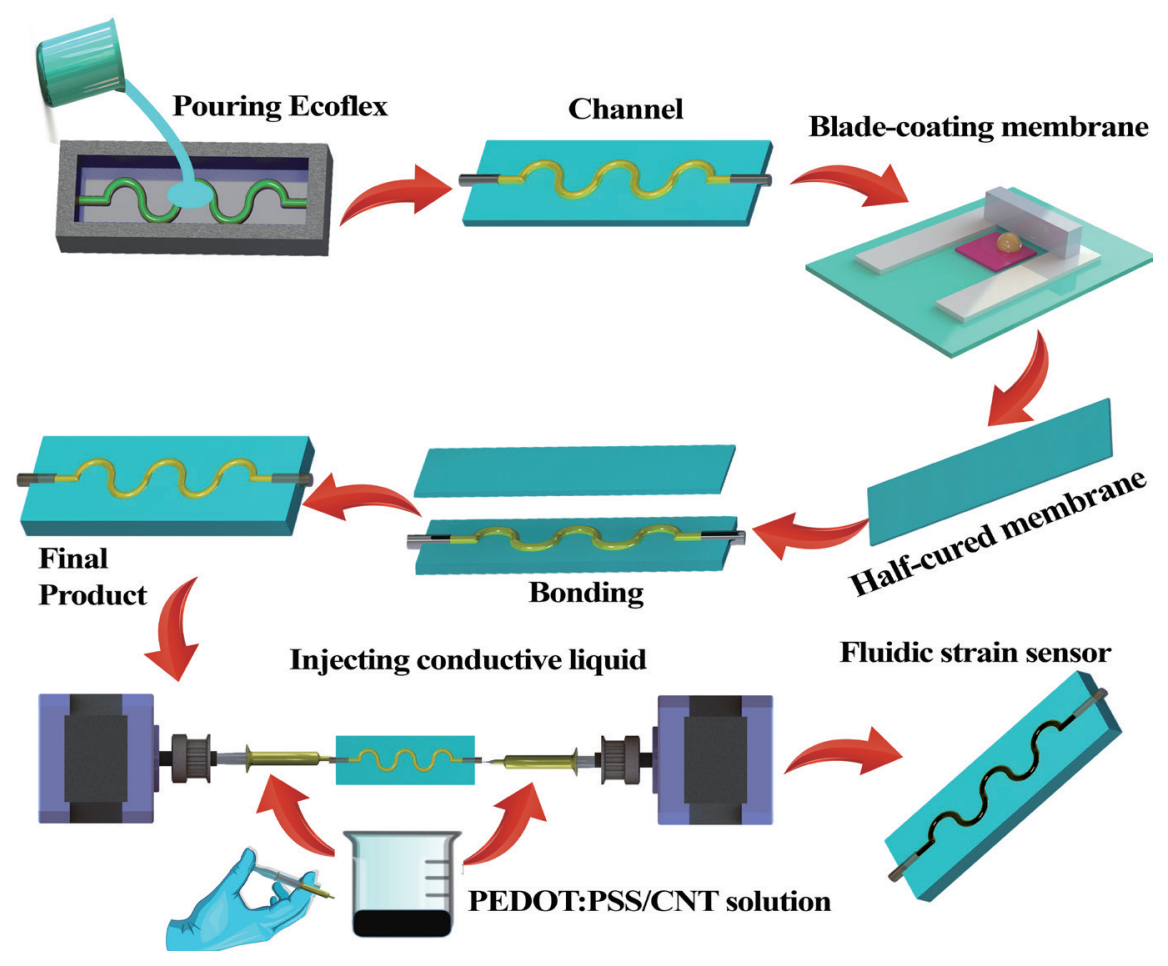

Fig. 1. (Color online) Sensor fabrication steps. 
prepared sensor with conductive liquid was physically deformed into different physical states, and optical images of these states are shown in Fig. S2.

\subsection{Strain-resistance characterization}

For the characterization of the fabricated fluidic strain sensor, a custom-built moving stage with the capability to apply uniaxial stretching and releasing strain was built on the sensor. The stage can be precisely actuated by a stepper motor with the help of an Arduino Mega and a motor driving circuit. Through serial communication via USB connection with a personal computer, various strain magnitudes and speeds can be applied. Figure 2(a) shows a schematic of the strain characterization setup. Moreover, optical images of the sensors under different stretching conditions $(0,50,100$, and 150\%) are shown in Fig. 2(b). The electrodes at both ends are used to measure the resistance of the sensor, which is directly related to the increase in length; there is an increase in resistance due to an increase in strain. For the measurement of strain, a portable meter (GW INSTEK LCR-916) was used. The meter was not only able to display real-time data, but also sent data to the computer serially, which was saved in a Microsoft excel file and later further analyzed. The sensor was characterized under roomtemperature conditions unless specifically indicated.
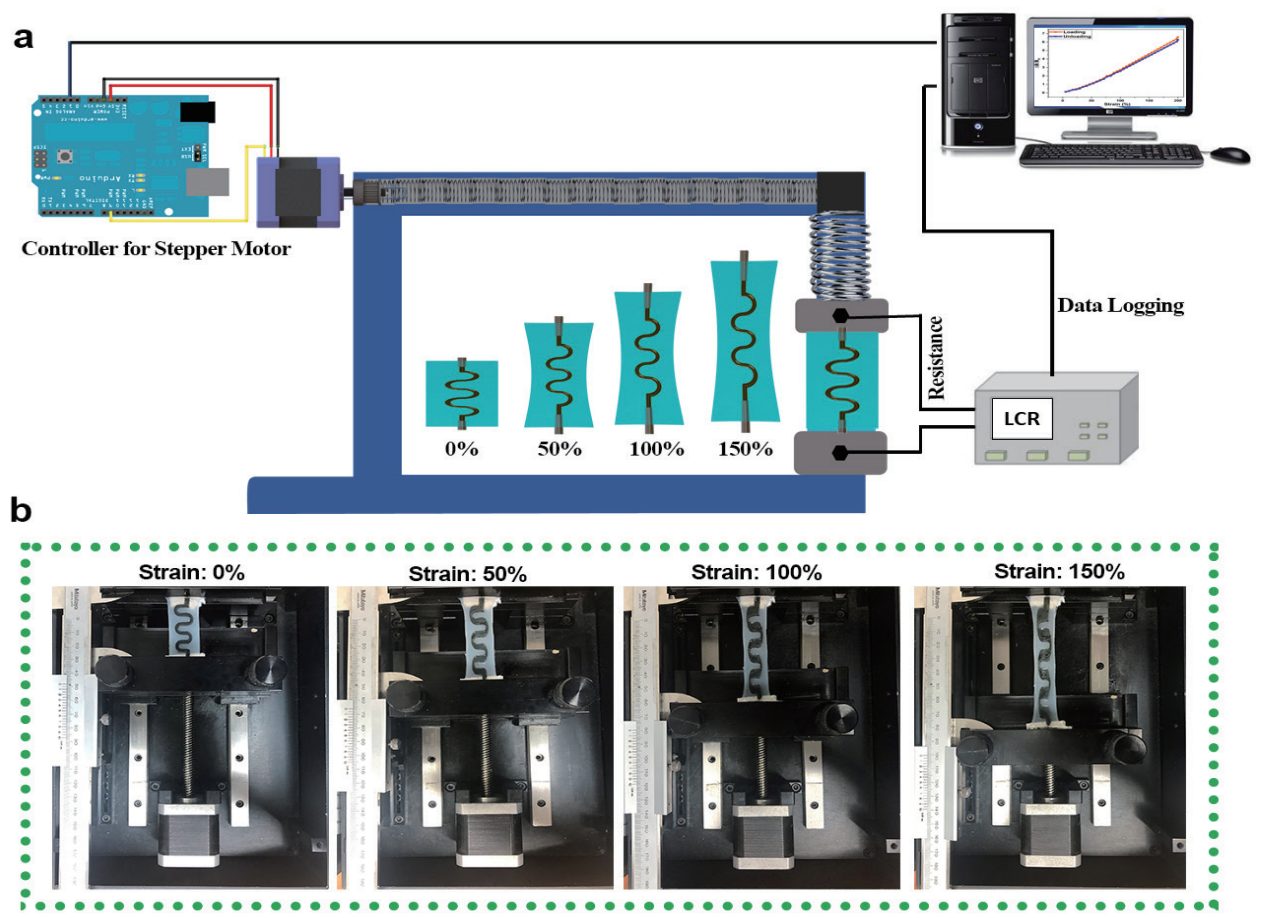

Fig. 2. (Color online) (a) Custom-made tensile strain characterization setup with capability of applying variable strain with programmed frequency and (b) optical images of sensor under different strain conditions $(0,50,100$, and $150 \%)$. 


\section{Results and Discussion}

\subsection{Sensor development process and working principle}

The fabrication of the proposed fluidic strain sensor followed a very simple process. First, a mold having a 2-mm-wide channel with a sinusoidal pattern was 3D-printed using a PLA filament $(1.75 \mathrm{~mm})$. The mold was then cast with Ecoflex 00-30 and left to cure at room temperature for $4 \mathrm{~h}$. For the matrix of the sensor, Ecoflex was selected owing to its ultrastretchable capability ( $900 \%$ strain), proven biocompatibility, and human-skin-like nature. For the complete encapsulation of the conductive liquid, the silicon-coated electrodes were attached to the mold in advance, which avoided the formation of tiny pores that could cause the leakage of the liquid. The cured elastomer was peeled off from the mold by applying a pulling force from all its sides. To apply a conformal cover to the channel where liquid was to be infilled, a thin membrane was fabricated by blade coating. However, the membrane was not intentionally fully cured; rather, it was half-cured by placing it at room temperature for $2 \mathrm{~h}$. Then, the same half-cured membrane was bonded to the channel matrix by placing it face down on the elastomer. Finally, the proposed conductive PEDOT:PSS/MWCNT solution was infilled to the channel using two syringes controlled by stepper motors; one syringe was used to pump in the conductive liquid and the other syringe was used to synchronously pump out the air from the channel.

The working principle of the proposed sensor is based on a simple concept: the resistance is directly proportional to the effective length of the conductive liquid. Herein, as strain is applied to the device, its length starts increasing, i.e., the length of the liquid leading to increased resistance across the two electrodes. The PEDOT:PSS/MWCNT composite was used owing to its high shuttling capacity of electrons inside the channel, Moreover, for strain-sensing applications, it is important to have a conductive polymer, which has the inherent feature of elasticity; PEDOT:PSS not only helps to minimize the hysteresis due to its faster conductive path recreations, but also retains the memory of its initial position at higher frequencies. The incorporation of MWCNTs further improves the conductivity, sensitivity, and memory effect in addition to strengthening the conductive articulated paths between the electrodes. The structure of the conductive liquid present in the channel is shown in Fig. 3(a). Moreover, the viscoelasticity of the elastomer also limits the ability of the sensors to achieve a better memory effect due to the imperfect attachment of the elastomer between sidewalls and conductive liquid molecules. This is a straight channel, wherein the elastomer volume $\left(V_{E C}\right)$ increases owing to stretching. Moreover, the Poisson ratio of elastomers is lower than 0.5 in practice. However, in the proposed sensor, the volumes of the conductive liquid and elastomeric channel remain constant. To confirm the volume limitation $\left(V_{E C}=V_{I L}\right)$, the channel is affected by compressing forces, enabling contraction in the axial direction. This contraction has been minimized by the design of the sinusoidal channel, which incorporates a sheer stress axial component and compressive force relaxation as can be seen in Fig. 3(b); this causes significantly a less lateral change in the length of the sensor. The nonuniform strain-stress distribution in the wavy channel enables the wavy channel to outperform a simple straight channel. ${ }^{(1,13)}$ Details of the 


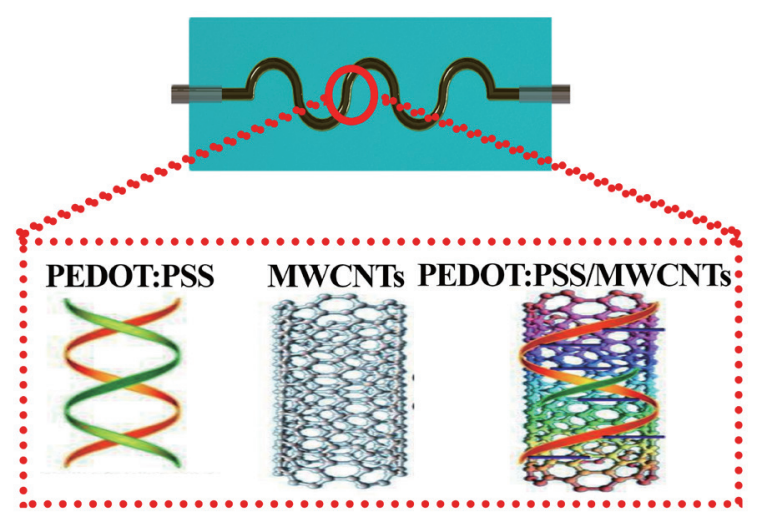

(a)

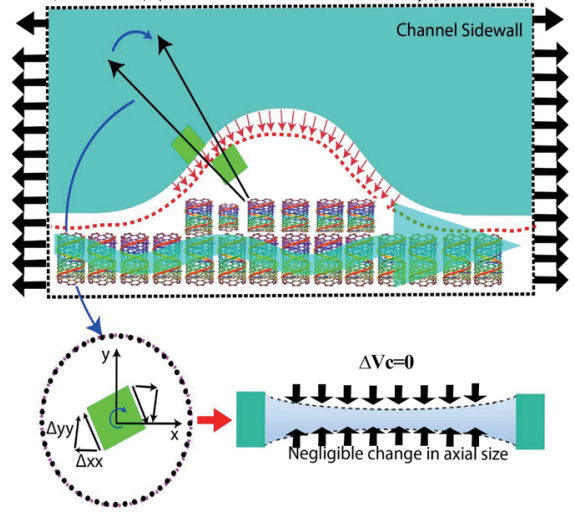

(b)

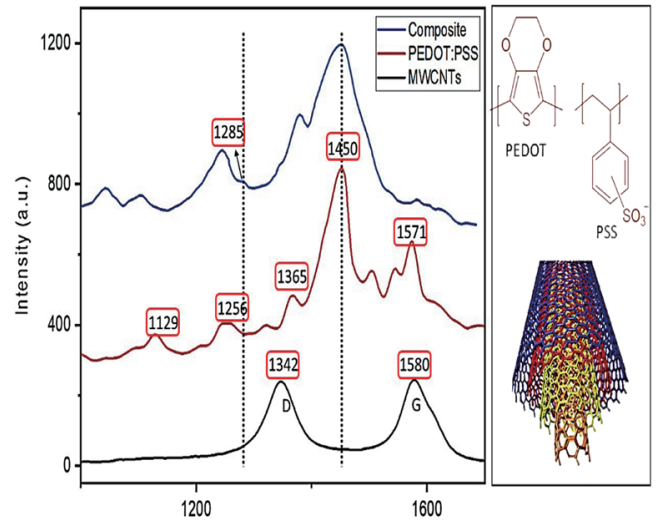

(c)

Fig. 3. (Color online) (a) Schematic showing structure of conductive PEDOT:PSS/MWCNT present in the channel. (b) Schematic showing a segment of the stretched sidewall of the infilled channel. Upon axial stretching, the effective length of the conductive liquid increases, whereas the relaxation is suppressed by the horizontal stress component. (c) Raman spectra of the composite of MWCNT and PEDOT:PSS, PEDOT:PSS, and the MWCNT.

concept of this design were reported in Ref. 5. Figure 3(c) shows the Raman spectra of the proposed composite and the individual MWCNT and PEDOT:PSS dispersions in DMF solvent (D, G, and 2D bands) at a resolution of $500 \mathrm{~nm}$. Moreover, two functions were considered while performing the numerical analysis of the proposed strain sensor: the bulk relaxation modulus $K(t)$ and shear modulus $G(t)$ given in Eqs. (1) and (2), respectively. Herein, $G_{0}$ and $K_{0}$ represent the time-dependent shear and bulk moduli, respectively, whereas the other parameters denote material coefficients.

$$
K(t)=G_{0} \sum_{i=1}^{n} g_{i}\left[\left(1-e^{-t / \partial_{i}}\right)\right]
$$




$$
G(t)=K_{0}\left[1-\sum_{i=1}^{n} k_{i}\left[\left(1-e^{-t / \partial_{i}}\right)\right]\right]
$$

The electrical characterization of the as-developed sensor was performed by measuring the output resistance across the two electrodes of the device against the applied uniaxial strain. The measured resistance was then converted to the relative change in resistance $\left(\Delta R / R_{0} \times 100\right)$. The sensor was tested in the strain range of $0-150 \%$. Figure 4(a) shows the relative resistance against the applied strain of the sensor. The strain was increased/decreased with a speed of $0.5 \mathrm{~Hz}$. Moreover, the hysteresis was calculated to be $1.56 \%$ using Eq. (1). ${ }^{(27)}$ There is a significantly higher consistency among the curves for stretching and releasing, which is associated with the inherent elasticity of PEDOT:PSS and the unique sinusoidal design of the channel. The minimized memory effect in the proposed sensor makes it a robust option for use in high-end feedback and a myriad of other relevant applications.

$$
D H=\frac{A_{\text {Loading }}-A_{\text {Unloading }}}{A_{\text {Loading }}} \times 100
$$

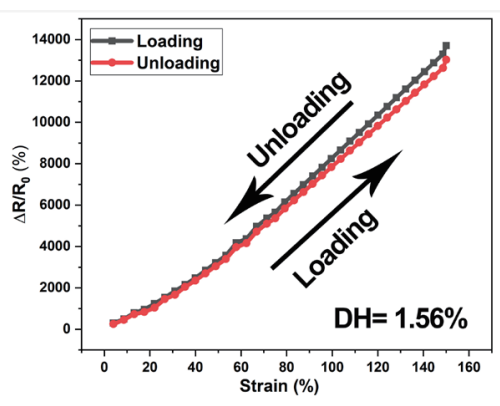

(a)

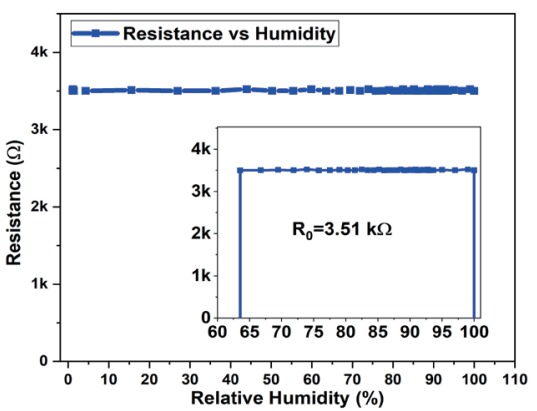

(d)

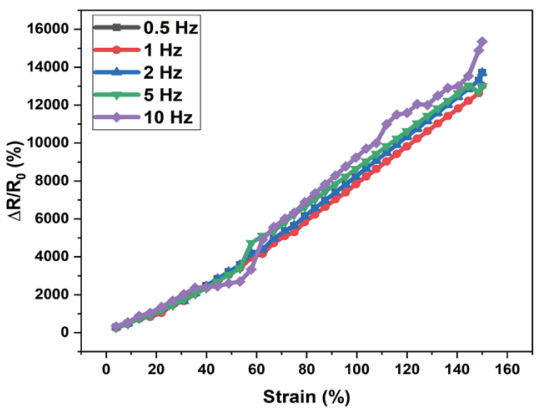

(b)

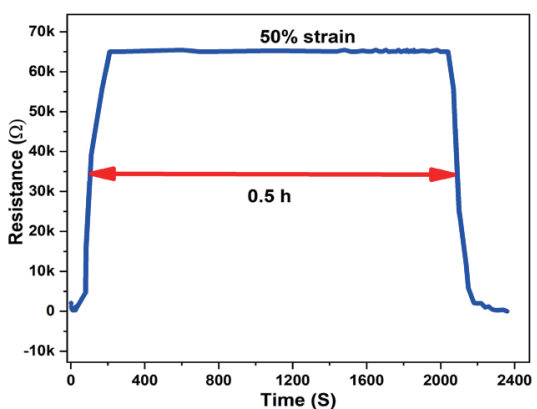

(e)

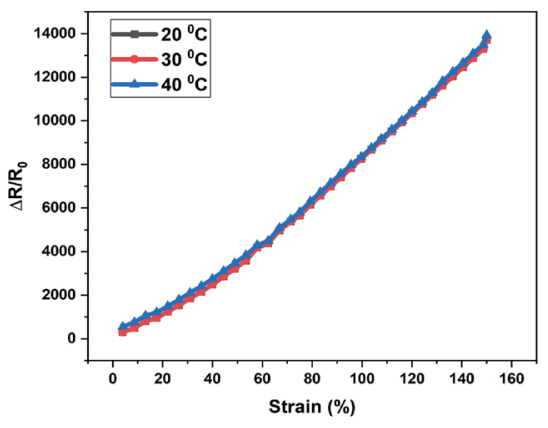

(c)

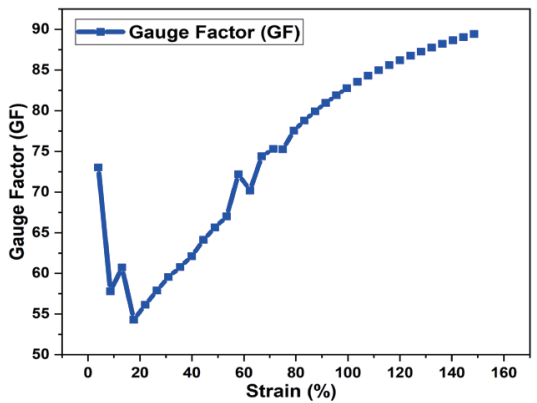

(f)

Fig. 4. (Color online) (a) Sensor loading/unloading response from 0 to 150 strain, (b) sensor response as a function of the applied frequency, (c) sensor response at temperatures of 20,30 , and $40{ }^{\circ} \mathrm{C}$, (d) sensor response under humid environment from 0 to $100 \% \mathrm{RH}$, (e) sensor response at $50 \%$ strain for $0.5 \mathrm{~h}$, and (f) $G F$ plotted against applied strain. 
It is essential that the strain sensor produces a consistent output resistance for various speed-driven strain applications. To experimentally show that the strain sensor possesses this property, the uniaxial strain was applied over the full range $(0-150 \%)$ at programmed speeds $(0.5$, 1, 2, 5, and $10 \mathrm{~Hz}$ ), the results of which are shown in Fig. 4(b). The device under test showed excellent consistency when operated at different frequencies. Moreover, at all speeds, the $G F$ was $~ 89.4$. There was a significant improvement in the performance of the proposed sensor at very low frequencies such as $0.5 \mathrm{~Hz}$ and at relatively high frequencies such as $10 \mathrm{~Hz}$, which makes it a favorable candidate for not only human body motion quantification (which requires a frequency of $2 \mathrm{~Hz}$ ) but also robotic applications (which can function at $10 \mathrm{~Hz}$ or more). ${ }^{(28)}$

Moreover, the sensitivity of the strain sensor plays a major role in its performance. To quantify this figure of merit, the $G F$ was calculated using Eq. (4). ${ }^{(29)}$ Herein, the changes in resistance and length are represented by $\Delta R$ and $\Delta L$, and $R_{0}$ and $L_{0}$ represent the initial resistance and length, respectively, when the sensor is under no strain condition. As shown in Fig. 4(f), the sensor achieved a maximum $G F$ of 89.4 at the maximum strain of $150 \%$, which is considerably higher than those of fluidic strain sensors reported in the literature, as shown in Table 1. This ultrasensitive feature of the proposed sensor is due to the higher conductivity of the conductive liquid, the optimized design, and the strong electrical paths augmented by MWCNTs. Moreover, there is some fluctuation in GF response below 20\% strain; this is due to the initial abrupt change in viscoelastic strain/stress, which causes breakdown in the electrical paths. However, despite this fact, the minimum $G F$ attained by the sensor was 55, which is itself a good performance figure of merit.

$$
G F=\frac{\left(\Delta R / R_{0}\right)}{\left(\Delta L / L_{0}\right)}
$$

The stability of the strain sensor plays a key role in high-end applications. The higher the stability, the more precise and accurate the decisions that can be taken using the sensor feedback. To confirm this, two different experiments were performed. First, a fixed strain

Table 1

Comparison of proposed sensor with previously reported fluidic strain sensors.

\begin{tabular}{lcccccccc}
\hline Sensing material & $\begin{array}{c}\text { Matrix } \\
\text { material }\end{array}$ & $\begin{array}{c}\text { Sensing } \\
\text { range }(\%)\end{array}$ & $\begin{array}{c}\text { Strain } \\
\text { frequency (Hz) }\end{array}$ & DH (\%) & GF & Reproducibility & COD & Ref. \\
\hline GaInSn & PDMS & 50 & - & - & - & 20 & Almost linear & $(30)$ \\
NaCl-Ecoflex & Ecoflex & 50 & - & 21.34 & - & - & $\sim 0.999$ & $(7)$ \\
Carbon grease solution & Ecoflex & 100 & - & 9.04 & 3.8 & 1000 & Nonlinear & $(31)$ \\
KI-Gly & Ecoflex & 50 & 2 & 5.3 & 2.2 & 4000 & Almost linear & $(32)$ \\
Ethylene glycol/NaCl & Ecoflex & 250 & - & 6.52 & $<4$ & 3000 & $\sim 0.989$ & $(14)$ \\
PEDOT: PSS & PDMS & 30 & - & $\sim 9$ & 12000 & - & - & $(33)$ \\
Graphene/glycerine & Ecoflex & 1000 & - & - & 45 & 10000 & Almost linear & $(25)$ \\
rGO/DI & Ecoflex & 400 & - & 31.6 & - & - & Almost linear & $(13)$ \\
KCL-Gly & Ecoflex & 100 & 5 & 4.23 & 2.7 & 8000 & $\sim 0.99$ & $(5)$ \\
PEDOT:PSS/MWCNT & Ecoflex & 150 & 10 & 1.56 & 89.4 & 1000 & 0.99 & This work \\
\hline
\end{tabular}


of $50 \%$ was applied across the device for $0.5 \mathrm{~h}$. Figure 4(e) shows the sensor response, which is highly stable. The resistance was measured to be $\sim 65 \mathrm{~K} \Omega$ at a uniaxial strain of $50 \%$; this remained constant throughout the experiment. Second, the device was tested for a long run (1000 cycles) at a strain of $30 \%$. The speed during this experiment was fixed to be $0.5 \mathrm{~Hz}$. The reproducibility of the sensor response is shown in Fig. 5(a). However, the responses considerably overlap owing to the display of 1000 cycles on the same graph, so the first and last 10 cycles are shown in Figs. 5(c) and 5(d), respectively. Despite the long run of the device, the response remained highly consistent, and little fluctuation can be seen in the results. This guarantees the strong tendency for the fabricated sensors to show a reproducible electrical response. Moreover, four different strains (25, 50, 100, and 150\%) were applied for 10 cycles across the device as shown in Fig. 5(b). The results are highly consistent and stable. In addition, every strain value is clearly distinguishable from those at other magnitudes. Similarly, three different sensor devices were prepared and characterized as a function of the applied strain. The results presented in Fig. 5(f) show excellent coherence and reproducibility, indicating the possibility of the mass production of the proposed sensor. Thus, we claim that the proposed sensor can be used in wearable and robotic applications, where high accuracy is required. The device response and recovery time were also calculated by applying stretching forces from 0 to $150 \%$ then removing the force and allowing it to return to its original position. Figure 5(e) shows that the as-developed sensor achieved very short response and recovery times of 720 and $900 \mathrm{~ms}$, respectively. The rapid sensing performance of the sensor paves the way for its efficient use in high-speed robotic applications more efficiently. The relationships between resistance and $G F$ is represented by Eq. (5), where $\gamma$ represents the contact resistance.

$$
\frac{\Delta R}{R_{0}}=\gamma\left(G F^{2}+2 G F\right)
$$

\subsection{Sensor response to humidity and temperature}

The stability of the sensor under humid conditions is essential; its response should not be affected by changes in atmospheric humidity. To confirm the waterproof feature and stability of the proposed fluidic strain sensor in a humid environment, we characterized its response over the entire relative humidity range $(0-100 \% \mathrm{RH})$ using our custom-designed computer-controlled chamber. For reference, a DTU-21D commercial sensor was used to measure the relative humidity, which was interfaced with an ESP232 board, and its data was serially logged to a computer, as shown in Fig. S3. The humidity of the chamber was gradually changed with the help of a proportional integrator and a differentiator (PID) controller. It can be seen in Fig. 4(d) that the sensor response is highly stable across the entire RH range. This implies that the proposed sensor is unaffected by environmental humidity conditions. Similarly, the fabricated device performance was analyzed while changing the temperature. The sensor and custombuilt stage were placed inside a temperature-controlled chamber in which the entire strain range from 0 to $150 \%$ was applied. As shown in Fig. 4(c), the experiment was performed at 20, 30, and $40{ }^{\circ} \mathrm{C}$, and little effect of temperature on the proposed sensor was observed, indicating its 


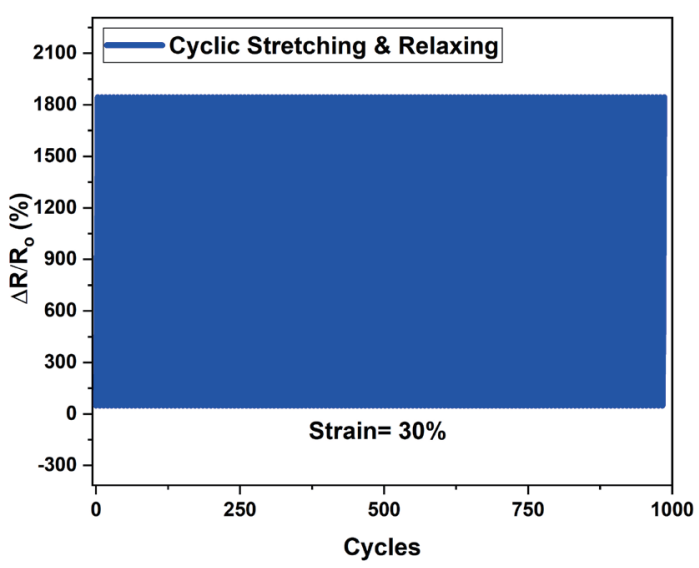

(a)

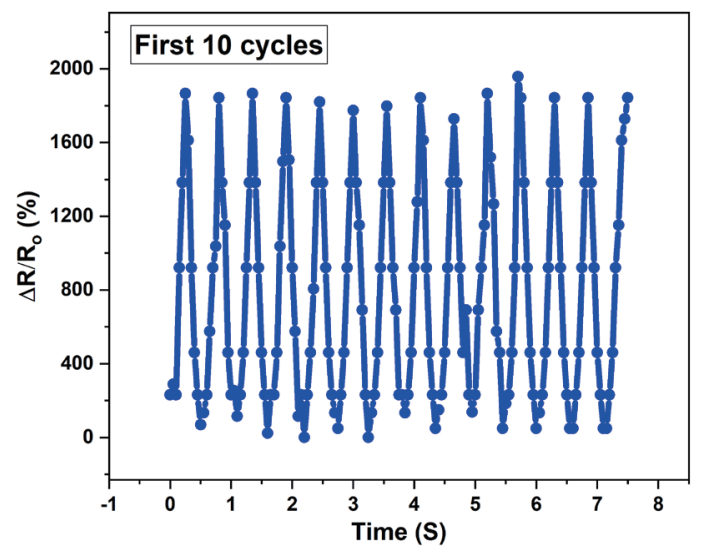

(c)

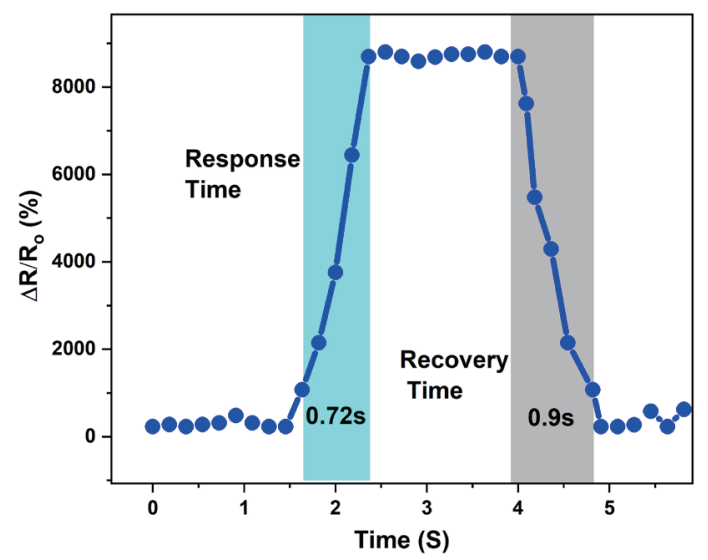

(e)

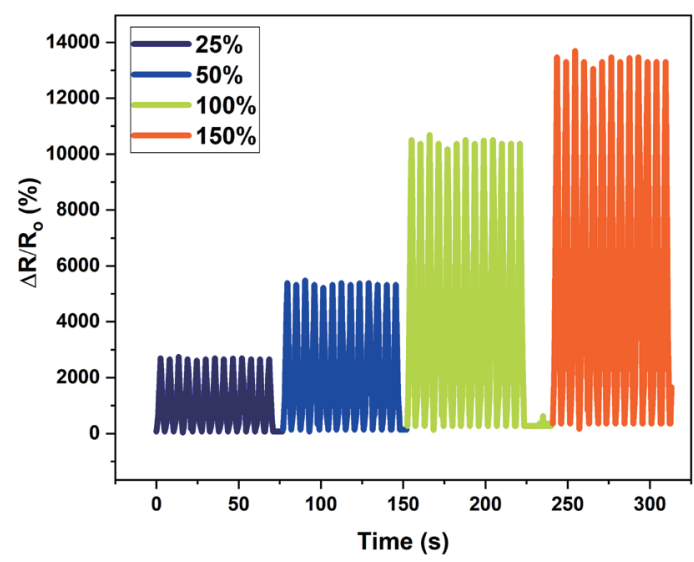

(b)

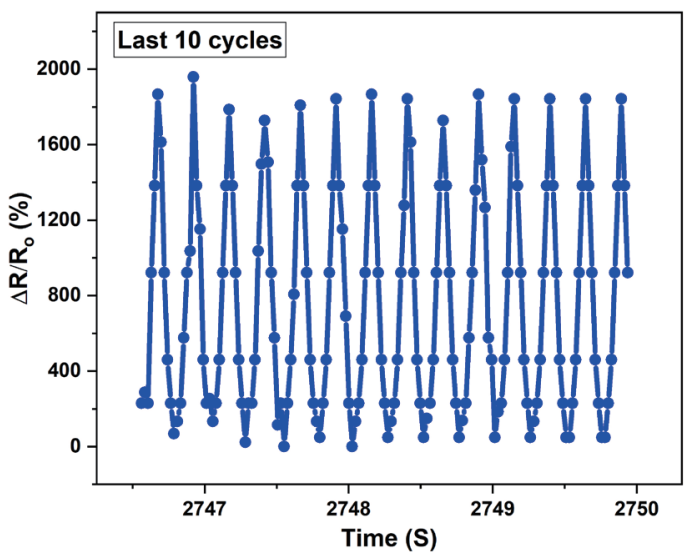

(d)

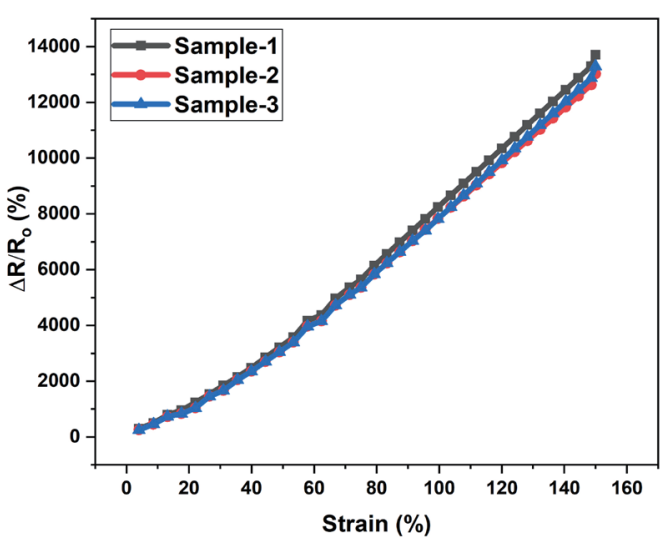

(f)

Fig. 5. (Color online) (a) Cyclic performance of sensor for 1000 cycles, (b) stability for 10 cycles under different strains, (c) first 10 cycles, (d) last 10 cycles, (e) sensor response and recovery times, and (f) response of three different samples plotted against strain. 
stability for various wearable health-monitoring applications where the temperature is around $40{ }^{\circ} \mathrm{C}$. This is also supported by the fact that the sensitivity of the sensor is not dependent on the conductivity, i.e., the relative change in resistance is independent of the conductive solution. ${ }^{(34)}$

\subsection{Biocompatibility test}

To target wearable applications for humans, it is of considerable importance that the proposed sensor must be biocompatible. This means that the sensor not only has high electrical and mechanical performance characteristics but also must not pose any harm to human skin when it is worn. To prove the biocompatibility of the developed sensor, we performed a live-dead assay experiment. For this, HEKp (Thermo Fisher Scientific) and HUVEC (ATCC) were cultured on a sensor surface made of PDMS at passage numbers 4 and 5, respectively. HEkp were cultured in serum-free mediaEpiLife ${ }^{\circledR}$ media with $60 \mu \mathrm{M}$ calcium and human keratinocyte growth supplement (HKGS), and HUVEC were cultured in a vascular cell basal medium with growth factors. Sensors containing cells were placed in petri plates with different media and kept at $37{ }^{\circ} \mathrm{C}$ in $5 \% \mathrm{CO}_{2}$ at $99 \%$ humidity for 7 days in an incubator. Cell viability was determined by a live/dead assay kit (Thermo Fisher). After 48, 96, and $156 \mathrm{~h}$, the sensors containing HEKp and HUVEC were washed with PBS and stained with $2 \mu 1$ of calcein to stain the incubated live cells for $20 \mathrm{~min}$ at room temperature and with $0.5 \mu \mathrm{l}$ of ethidium homodimer to stain the dead cells for $10 \mathrm{~min}$ at room temperature. They were then washed thrice and visualized under a confocal laser scanning microscope. Z-stack images were taken for all the samples.

Human primary skin and blood capillary cells were used to test the biocompatibility of the strain sensor. Live/dead assay was performed to examine the cell viability over a week. Our results showed good cell viability over a prolonged duration. The sensor did not show cytotoxicity to human skin or blood capillary cells even after 1 week of cell culture on the sensor surface. The survival rates of HEKp and HUVEC were $96.7 \pm 1.5 \%$ and $97.4 \pm 0.9 \%$, respectively, as shown in Fig. 6 .

\subsection{Application demonstrations}

The fabricated sensor was used in different wearable electronics and robotic applications for the measurement of human body motion and angle measurement, respectively. In the first proof-of-concept demonstration, the fabricated device was attached to the upper side of the index finger of a human's right hand using adhesive bandages, while thin and lightweight electrical wires were used to read out the device electrical resistance. The subject was free to bend the finger as desired. The experiment was divided into two movements: slow bending and fast bending. Moreover, before data logging, it was ensured that the output resistance of the sensor was stable $(\sim 3.51 \mathrm{~K} \Omega)$. Figure 7 (a) shows the results of the movement of the finger at room temperature and normal humidity $(\sim 40 \%)$. Slow bending and fast bending are clearly distinguishable, further confirming that the sensor response effectively follows the motion of the human finger. As soon as the finger is bent, the relative change in resistance jumps to a value of around $300 \%$. Similarly, it is important to monitor the mechanical movement of the human 

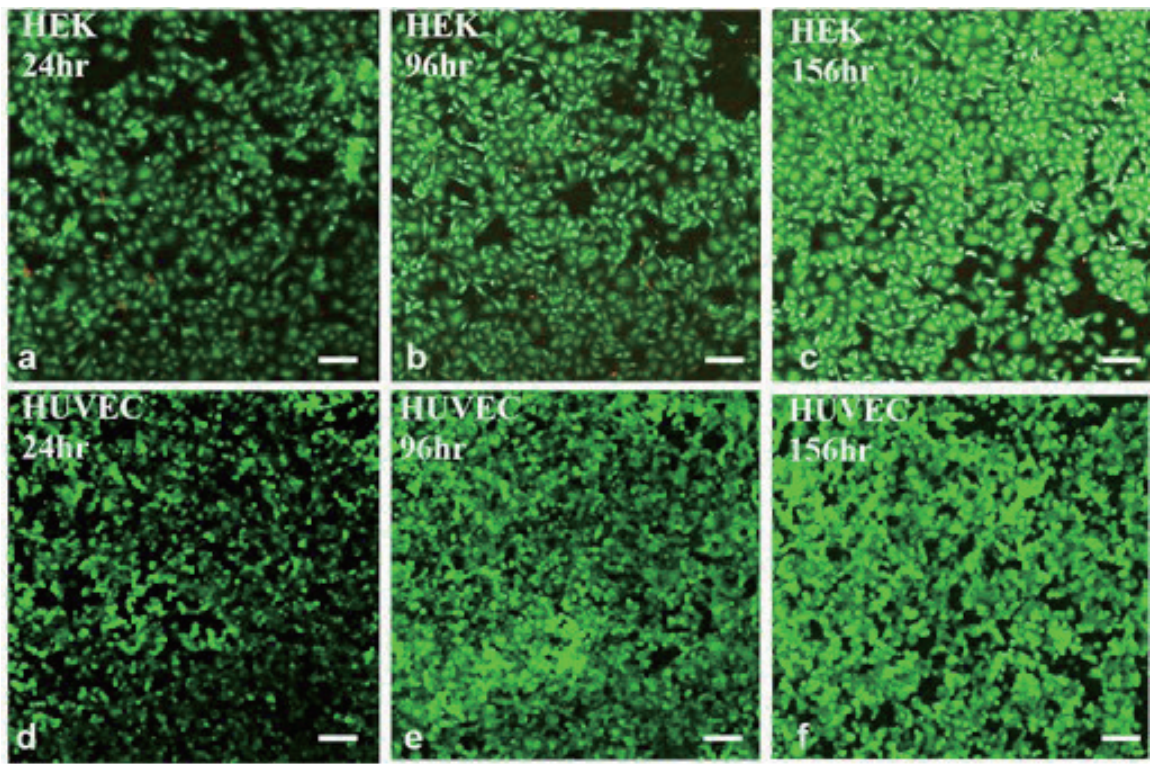

Fig. 6. (Color online) $(\mathrm{a}-\mathrm{c})$ Confocal laser scanning microscopy $\mathrm{z}$-stack images of HEKp increasing viability on the strain sensor after 48, 96, and $156 \mathrm{~h}$ [green fluorescence shows live cells, whereas red fluorescence shows dead cells; scale bar, $100 \mu \mathrm{m}(n=3)]$. $(\mathrm{d}-\mathrm{f})$ Confocal laser scanning microscopy z-stack images of HUVEC cells showing increasing viability on strain sensor after 48, 96, and $156 \mathrm{~h}$ [green fluorescence shows live cells, whereas red fluorescence shows dead cells; scale bar, $100 \mu \mathrm{m}(n=3)]$.

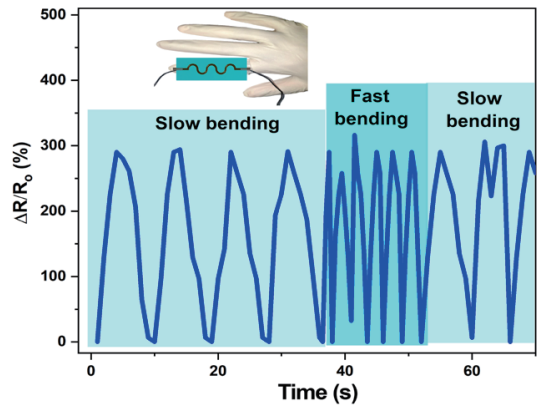

(a)

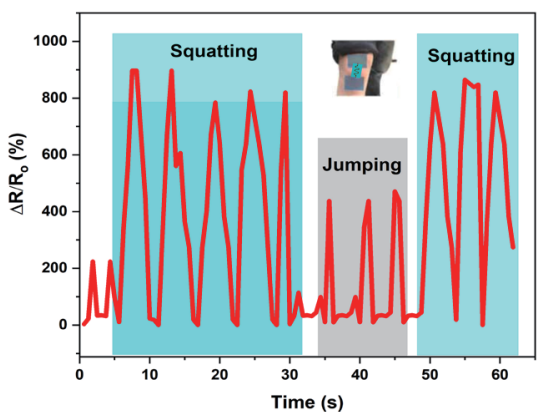

(d)

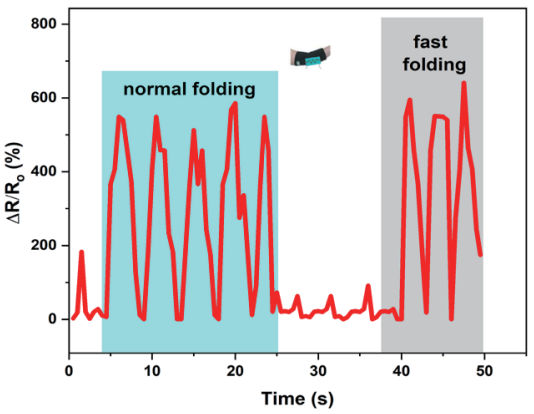

(b)

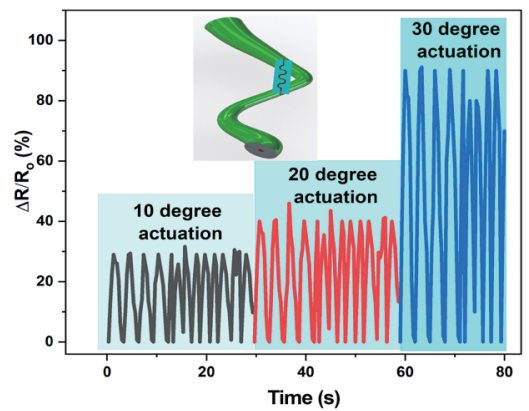

(e)

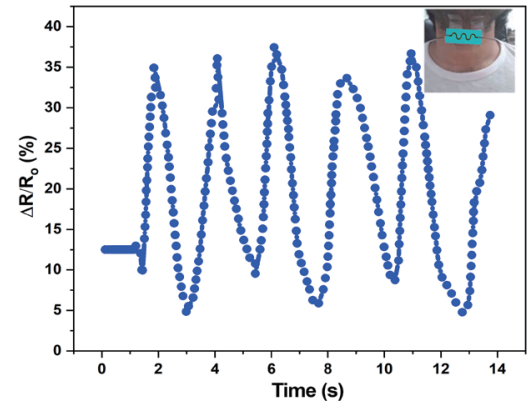

(c)

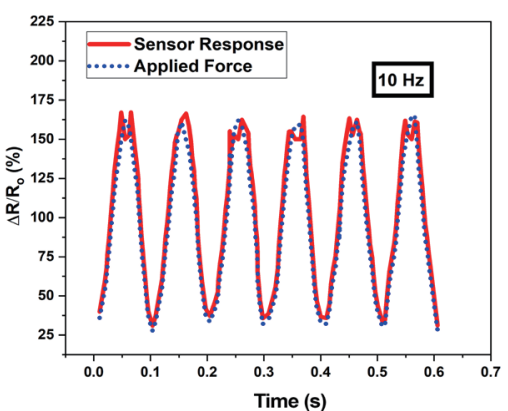

(f)

Fig. 7. (Color online) (a) Monitoring of man finger motion, (b) sensor response when attached on human elbow joint (c) monitoring of water drinking pattern, (d) sensor response when attached on knee joint, (e) sensor response when used with 3D-printed robotic leg, and (f) sensor response when strain with a speed of $10 \mathrm{~Hz}$ is applied. 
arm, especially in the case of fracture. To target this application, the device was wrapped around the elbow, which was at the center of the sensor. The data logged by the LCR meter is shown in Fig. 7(b). Similarly to the experiment on the finger, the subject was instructed to freely move his elbow at either a normal speed or a high speed as desired. The sensor showed responses of $590 \%$ and $10 \%$ under the bending and straightening conditions, respectively. The as-developed fluidic strain sensor clearly distinguished the two movements without any unit of precalibration or pretreatment. Moreover, to prove that the proposed sensor can measure small changes in physical deformations, the device was attached to the middle of the subject's neck. The subject was asked to drink water and the electrical response of the strain sensor was measured in real time, as shown in Fig. 7(c). Herein, the relative change in resistance (6-30\%) clearly indicates the drinking style of the subject. Moreover, the proposed device is promising for studying human drinking styles for various liquids.

The proposed sensor was next attached to a knee joint to monitor two different motion styles of a human subject: squatting and jumping. Long, thin, and light wires were used to connect the sensor electrode and measuring meter to gain realistic data. As shown in Fig. 6(d), the relative change in resistance attained a value of $\sim 800 \%$ as the subject started squatting, while in case of a relaxed straight posture, there was no strain on the device, resulting in a low resistance output. In the case of jumping, there was a sudden change in strain over the device, causing the change in resistance to reach $\sim 400 \%$. Moreover, the few small fluctuations in the readout were due to abrupt changes in the subject's physical posture.

Finally, the sensor was used as a high-end feedback sensor for a robotic leg. For this, a leg mimicking that of a frog was 3D-printed using a commercial printer. An Ultraflex PLA filament was used to print the soft leg of the frog. The actuation of the leg was made possible by embedding shape memory alloy (SMA) functional wires $(0.3 \mathrm{~mm}$ diameter). Upon the application of voltage $(5 \mathrm{~V})$, a current of around 1 A started flowing, which heated the wires, resulting in the contraction of the leg. However, to optimize the performance of the sensor to the leg, three angular movements $\left(10,20\right.$, and $\left.30^{\circ}\right)$ of the leg were precalibrated using electricalcurrent optimization methods. Figure 7(e) shows the response of the sensor to the movements over multiple cycles. The device response is highly distinguishable for each movement. The change in electrical resistance at $30^{\circ}$ actuation was more than double at $10^{\circ}$ actuation. The actuation speed was limited by the cooling/heating of the SMA-embedded robotic leg. However, to further prove that the device can even be used for relatively high frequency applications in robotics, we applied a sinusoidally governed strain to the custom-made stage used to uniaxially deform the fabricated device at two frequencies $(5$ and $10 \mathrm{~Hz}$ ). The responses of the sensor at 5 and $10 \mathrm{~Hz}$ are given in Figs. S4 and 7(f), respectively. The device closely follows the applied sinusoidal pattern of the strain at the very higher frequency of $10 \mathrm{~Hz}$. This proves that the proposed sensor can be a robust option for high-end robotic feedback application, in addition to personal wearable electronics.

\section{Conclusion}

We have designed an ultraflexible, highly stable, and linear fluidic strain sensor using a biocompatible conductive composite based on PEDOT:PSS/MWCNT. The inherent elastic 
property of PEDOT:PSS and the excellent conductivity of MWCNTs enable the sensor to attain a two-order change in electrical resistance under the maximum strain of $150 \%$. The fabricated device achieved excellent performance in hysteresis $(1.56 \%)$, high linearity $\left(R^{2}=\right.$ 0.9935), and sensitivity with a $G F$ of 89.4 at a strain of $150 \%$. The sensor showed a high level of reproducibility for 1000 cycles at a strain of $30 \%$ and consistency in precisely following the applied strain at a speed of $10 \mathrm{~Hz}$. The device was tested at different temperatures (20 to $40{ }^{\circ} \mathrm{C}$ ) and humidities (0-100\% RH). The biocompatibility of the sensor was also confirmed by culturing HEKp and HUVEC using the live/dead assay method. As a proof-of-concept demonstration, various wearable and robotic feedback applications such as the monitoring of robotic leg, human finger, human arm, human knee joint, and human neck movements were targeted. The results confirm that the proposed sensor with a simple fabrication process is robust for high-end feedback applications.

\section{Conflict of Interest}

The authors declare no conflict of interest.

\section{Acknowledgments}

This work was supported by the National Research Foundation of Korea (NRF) Grant funded by the Korea Government (MSIT) (NRF-2018R1A2B3001830).

\section{References}

1 C. H. Lee, Y. Jeon, and H. Ashraf: IEEE Access 8 (2020) 182193. https://doi.org/10.1109/ ACCESS.2020.3028134

2 H. Ashraf, Y. Jeong, and C. H. Lee: J. Acoust. Soc. Kor. 4 (2020) 292. https://doi.org/10.7776/ ASK.2020.39.4.2923

3 J. Luan, Q. Wang, X. Zheng, Y. Li, and N. Wang: Micromachines 10 (2019) 6. https://doi.org/10.3390/ mi10060372

4 A. M. Soomro, F. Jabbar, M. Ali, J.-W. Lee, S. W. Mun, and K. H. Choi: J. Mater. Sci. Mater. Electron. 30 (2019) 9455. https://doi.org/10.1007/s10854-019-01277-1

5 A. M. Soomro, M. A. U. Khalid, I. Shah, S. W. Kim, Y. S. Kim, and K. H. Choi: Smart Mater. Struct. 29 (2020) 2. https://doi.org/10.1088/1361-665x/ab540b

6 M. A. U Khalid, M. Ali, A. M. Soomro, S. W. Kim, H. B. Kim, B. Leeb, and K. H. Choi: Sens. Actuators, A 294 (2019) 140. https://doi.org/10.1016/j.sna.2019.05.021

7 S. Russo, T. Ranzani, H. Liu, S. Nefti-Meziani, K. Althoefer, and A. Menciassi: Soft Robot. 2 (2015) 4 . https:// doi.org/10.1089\%2Fsoro.2015.0011

8 J. W. Lee, A. M. Soomro, M. Waqas, M. A. U. Khalid, and K. H. Choi: Int. J. Energy Res. 44 (2020) 8. https:// doi.org/10.1002/er.5371

9 C. H. Lee, Y. S. Jeong, and H. Ashraf: Sensors 19 (2019) 21. https://doi.org/10.3390\%2Fs19214626

10 F. Yin, J. Yang, H. Peng, and W. Yuan: J. Mater. Chem. C 6 (2018) 25. https://doi.org/10.1039/C8TC00839F

11 T. Zhou, B. Guo, and J. Xu: Adv. Mater. Technol. 2000508 (2020) 1. https://doi.org/10.1002/admt.202000508

12 M. Bhattacharjee, M. Soni, P. Escobedo, and R. Dahiya: Adv. Electron. Mater. 6 (2020) 200445. https://doi. org/10.1002/aelm.202000445

13 M. Xu, J. Qi, F. Li, and Y. Zhang: Nanoscale 10 (2018) 11. https://doi.org/10.1039/C7NR09022F

14 D. Y. Choi, S. H. Jung, D. K. Song, E. J. An, D. Park, T. Kim, J. H. Jung, and H. M. Lee: ACS Appl. Mater. Interfaces 9 (2017) 2. https://doi.org/10.1021/acsami.6b12415

15 Y. Cheng, R. Wang, J. Sun, and L. Gao: Adv. Mater. 27 (2015) 45. https://doi.org/10.1002/adma.201503558 
16 M. Waqas, A. M. Soomro, and S. Ahmed: Chemselect. 5 (2020) 12009. https://doi.org/10.1002/slct.202003381

17 J. Herrmann, K.-H. Müller, T. Redab, G. R. Baxter, B. Raguse, G. J. J. B. de Groot, R. Chai, M. Roberts, and L. Wieczorek: Appl. Phys. Lett. 91 (2007) 18. https://doi.org/10.1063/1.2805026

18 A. Nakamura, T. Hamanishi, S. Kawakami, and M. Takeda: Mater. Sci. Eng. B Solid-State Mater. Adv. Technol. 219 (2017) 20. https://doi.org/10.1016/j.mseb.2017.02.012

19 J. L. Tanner, D. Mousadakos, K. Giannakopoulos, E. Skotadis, and D. Tsoukalas: Nanotechnology 23 (2012) 28. https://doi.org/10.1088/0957-4484/23/28/285501

20 Y. Huang, X. Guo,Y. Zhao, L. Mao, L. Gao, W. Pan, Y. Zhang, and P. Liu: Smart Mater. Struct. 26 (2017) 9. https://doi.org/10.1088/1361-665x/aa79c3

21 V. Arabagi, O. Felfoul, A. H. Gosline, R. J. Wood, and P. E. Dupont: IEEE Sens. J. 16 (2016) 5. https://doi. org/10.1109\%2FJSEN.2015.2498481

22 X. Shi, C. H. Cheng, Y. Zheng, and P. K. A. Wai: J. Micromech. Microeng. 26 (2016) 10. https://doi. org/10.1088/0960-1317/26/10/105020

23 S. Zhu, J. H. So, R. Mays, S. Desai, W. R. Barnes, and B. P. M. Dickey: Adv. Funct. Mater. 23 (2013) 18. https://doi.org/10.1002/adfm.201202405

24 J. Shintake, E. Piskarev, S. H. Jeong, and D. Floreano: Adv. Mater. Technol. 3 (2018) 3. https://doi.org/10.1002/ admt.201700284

25 Z. Qi, H. Bian, Y. Yang, N. Nie, and F. Wang: Nanomaterials 9 (2019) 4. https://doi. org $/ 10.3390 \% 2$ Fnano9040617

26 S. H. Zhang, F. X. Wang, J. J. Li, H. D. Peng, J. H. Yan, and G. B. Pan: Sensors 17 (2017) 11. https://doi. org $/ 10.3390 / \mathrm{s} 17112621$

27 P. Luo, F. Zhuge, Q. Zhang, Y. Chen, L. Lv, Y. Huang, H. Lia, and T. Zhai: Nanoscale Horizons 4 (2019) 26, 2019.

28 H. J. Freund: Prog. Brain Res. 64 (1986) 287.

29 S. Park, S. Ahn, J. Sun, D. Bhatia, D. Choi, K. S.Yang, J. Bae, and J. J. Park: Adv. Funct. Mater. 29 (2019) 1.

30 R. Matsuzaki and K. Tabayashi: Adv. Funct. Mater. 25 (2015) 25. https://doi.org/10.1002/adfm.201501396

31 J. T. Muth, D. M. Vogt, R. L. Truby, Y. Mengüç, D. B. Kolesky, R. J. Wood, and J. A. Lewis : Adv. Mater. 26 (2014) 36. https://doi.org/10.1002/adma.201400334

32 S. Xu, D. M. Vogt, W.-H. Hsu, J. Osborne, T. Walsh, J. R. Foster, S. K. Sullivan, V. C. Smith, A. W. Rousing, E. C. Goldfield, and R. J. Wood: Adv. Funct. Mater. 29 (2019). https://doi.org/10.1002/adfm.201970038

33 M. Bhattacharjee, M. Soni, P. Escobedo, and R. Dahiya: Adv. Electron. Mater. 2000445 (2020). https://doi. org/10.1002/aelm.202000445

34 S. Xu, D. M. Vogt, W.-H. Hsu, J. Osborne, T. Walsh, J. R. Foster, S. K. Sullivan, V. C. Smith, A. W. Rousing, E. C. Goldfield, and R. J. Wood: Adv. Funct. Mater. 29 (2019). https://doi.org/10.1002/adfm.201807058

\section{Supplement}

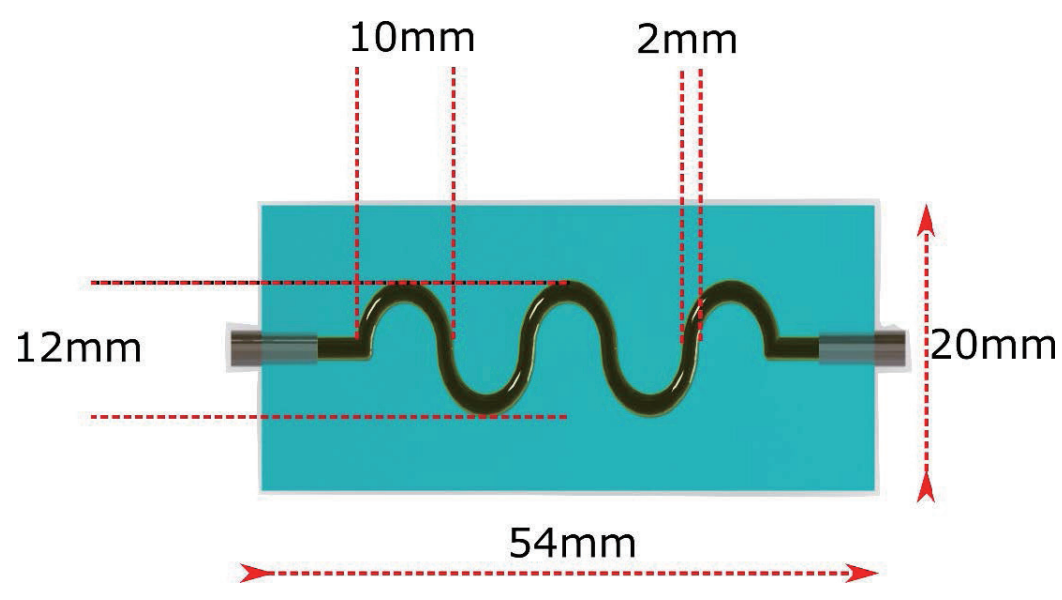

Fig. S1. (Color online) Dimensions of fabricated sensor. 

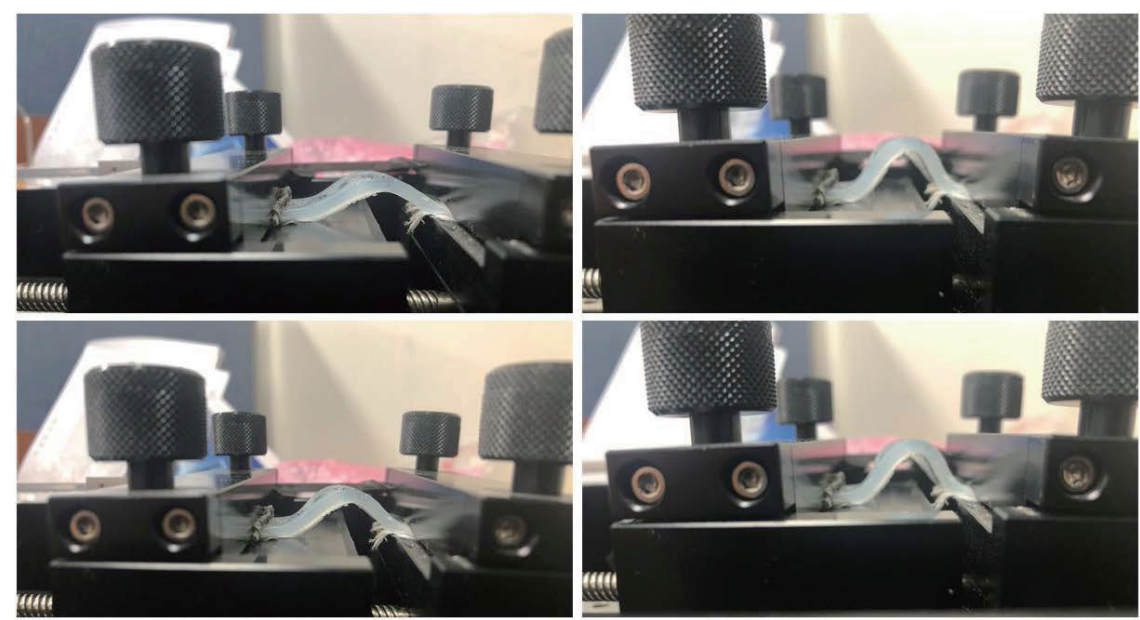

Fig. S2. (Color online) Optical images of sensor in various physical bending states.

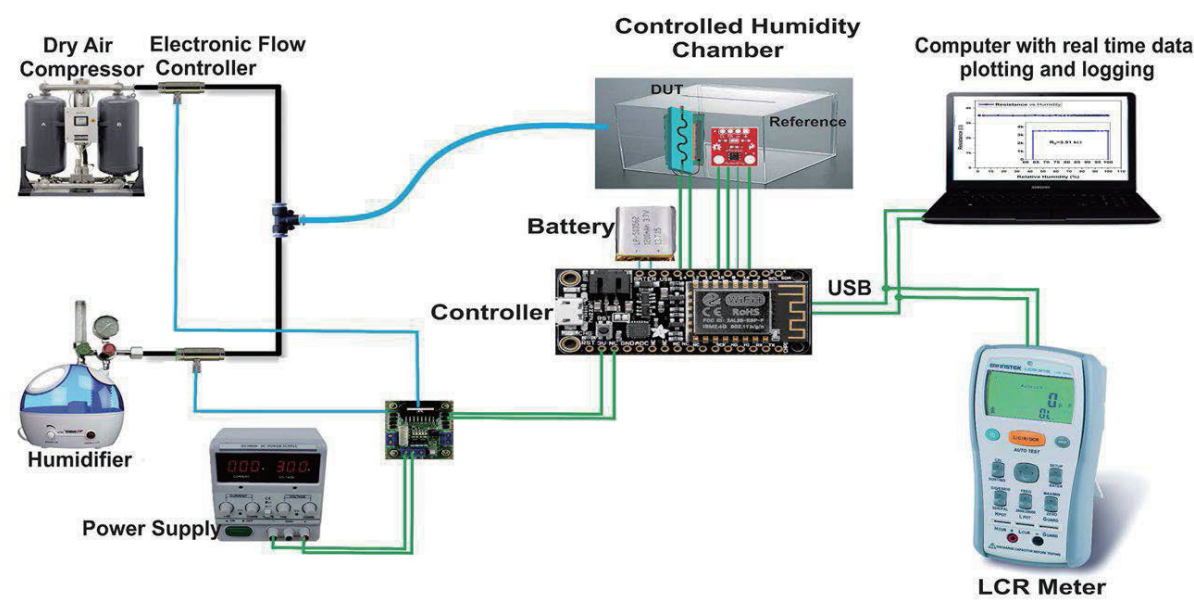

Fig. S3. (Color online) Setup schematic for sensor's characterization under humidity-controlled environment.

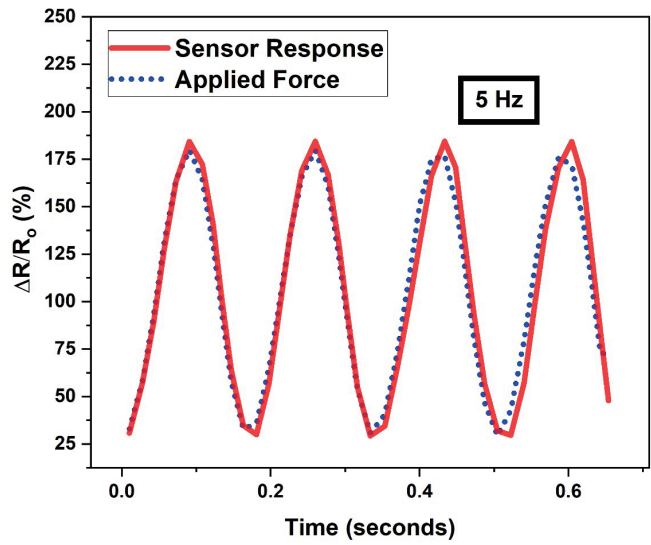

Fig. S4. (Color online) Sensor's response when strain with a speed of $10 \mathrm{~Hz}$ is applied. 
